\title{
Variability-selected active galactic nuclei from supernova search in the Chandra deep field south ${ }^{\star}$
}

\author{
D. Trevese ${ }^{1}$, K. Boutsia ${ }^{1,2}$, F. Vagnetti ${ }^{3}$, E. Cappellaro ${ }^{4}$, and S. Puccetti ${ }^{5}$
}

\author{
1 Dipartimento di Fisica, Università di Roma "La Sapienza”, P.le A. Moro 2, 00185 Roma, Italy \\ e-mail: dario.trevese@roma1.infn.it \\ 2 European Southern Observatory, Karl-Schwarzschild-Strasse 2, Garching 85748, Germany \\ 3 Dipartimento di Fisica, Università di Roma "Tor Vergata", via delle Ricerca Scientifica 1, 00133 Roma, Italy \\ 4 INAF - Osservatorio Astronomico di Padova, Vicolo dell'Osservatorio 5, 35122 Padova, Italy \\ 5 ASI Science Data Centre, c/o ESRIN, via G. Galilei, 00044 Frascati, Italy
}

Received 1 April 2008 / Accepted 13 May 2008

\section{ABSTRACT}

\begin{abstract}
Context. Variability is a property shared by virtually all active galactic nuclei (AGNs), and was adopted as a criterion for their selection using data from multi epoch surveys. Low Luminosity AGNs (LLAGNs) are contaminated by the light of their host galaxies, and cannot therefore be detected by the usual colour techniques. For this reason, their evolution in cosmic time is poorly known. Consistency with the evolution derived from X-ray detected samples has not been clearly established so far, also because the low luminosity population consists of a mixture of different object types. LLAGNs can be detected by the nuclear optical variability of extended objects.

Aims. Several variability surveys have been, or are being, conducted for the detection of supernovae (SNe). We propose to re-analyse these SNe data using a variability criterion optimised for AGN detection, to select a new AGN sample and study its properties.

Methods. We analysed images acquired with the wide field imager at the $2.2 \mathrm{~m} \mathrm{ESO/MPI} \mathrm{telescope,} \mathrm{in} \mathrm{the} \mathrm{framework} \mathrm{of} \mathrm{the} \mathrm{STRESS}$ supernova survey. We selected the AXAF field centred on the Chandra Deep Field South where, besides the deep X-ray survey, various optical data exist, originating in the EIS and COMBO-17 photometric surveys and the spectroscopic database of GOODS.

Results. We obtained a catalogue of 132 variable AGN candidates. Several of the candidates are X-ray sources. We compare our results with an HST variability study of X-ray and IR detected AGNs, finding consistent results. The relatively high fraction of confirmed AGNs in our sample (60\%) allowed us to extract a list of reliable AGN candidates for spectroscopic follow-up observations.
\end{abstract}

Key words. surveys - galaxies: active - galaxies: quasars: general - X-rays: galaxies

\section{Introduction}

The optical variability of quasars (QSOs) was discovered even before the very nature of their emission lines was understood (Matthews \& Sandage 1963). A sharp decline in cosmic time of both radio- and optically-detected QSO populations was established soon after their discovery (Schmidt 1968, 1970). To our knowledge, the use of variability as a tool to detect QSOs was proposed for the first time by van den Berg et al. (1973). Any of the QSO properties, such as non-stellar colour, broad emission lines, or variability, can be used to select statistically relevant QSO samples. The comparison between samples selected using different techniques enables to evaluate the relevant selection effects and to derive the intrinsic cosmological evolution of the QSO population. Since the early days of QSO astronomy, we have learned that different techniques can detect different but related classes of objects; this occurred for the UV excess selection technique, and led to the discovery of radio-quiet QSOs, which are 5-10 times more numerous than radio-loud ones (e.g. Kellermann et al. 1989; Jiang et al. 2007). The present knowledge of the evolution with cosmic time of the QSO luminosity function (LF) is based mainly on the 2QZ survey (Boyle et al. 2000; Croom et al. 2001) for $z<2.5$, on spectroscopic surveys

* Table 2 is only available in electronic form at http: //www . aanda.org for $z \geq 3$ (Warren et al. 1994; Schmidt et al. 1995), and on Sloan Digital Sky Survey (SDSS) data for $z>4.5$ (Fan et al. 2001; Anderson et al. 2001). These analyses led to a consensus scenario where a rapid increase in the QSO number density with cosmic time, down to $z \sim 2$, is followed by a slower decline in the LF, which can be described by luminosity evolution. This view was confirmed by Richards et al. (2006), whose analysis, however, is not the best suited to locate the epoch at which the number density of active galactic nuclei (AGNs) reached a peak. Both 2QZ and SDSS QSO candidates are selected on the basis of non-stellar colours. This selection technique is limited to pointlike objects, i.e. bright active nuclei outshining the host galaxy, which, would otherwise produce non-stellar colours. The most accurate determination of the epoch of maximum density was determined by Wolf et al. (2003), on the basis of the COMBO17 survey, which provided a "low resolution spectrum" enabling the "point-like" condition to be neglected, for selecting AGNs on basis of their SED alone. The analysis by Wolf et al. (2003) indicates that the epoch of maximum density corresponds to $z \simeq 2$ and it is independent of QSO luminosity. Even in this case, however, the selection is limited to nuclei brighter than $M_{\mathrm{B}} \simeq-21.5$, since otherwise the SED is dominated by the host galaxy light, which prevents the nuclear spectrum from being recognised. Since van den Berg et al. (1973), variability was adopted as a tool for selecting QSOs/AGNs in various studies 
(Usher 1978; Hawkins 1983; Cristiani et al. 1990; Trevese et al. 1989; Véron \& Hawkins 1995; Geha et al. 2003). An important aspect of variability as an AGN search technique is that it can be applied to extended objects. These include low luminosity AGNs (LLAGNs) that cannot be detected by the (non-stellar) colour selection since their SED is contaminated (or even dominated) by the light of the host galaxy. In this case, variability selection becomes easier since nuclear variability tends to increase as nuclear luminosity decreases (Hook et al. 1994; Trevese et al. 1994; Cristiani et al. 1996). Bershady et al. (1998) selected a sample of "variable galaxies", i.e. galaxies with variable nuclei, in the field of Selected Area 57, where other detection techniques had been applied: these techniques included the use of colours and absence of proper motion (Koo et al. 1986; Koo \& Kron 1988), and for several objects the AGN character was demonstrated either by spectroscopic observations (Trevese et al. 2008) or by X-ray emission (Trevese et al. 2007) (see below).

In many respects, the best way of detecting AGNs is just to use X-ray surveys, which enable us to distinguish between stellar and accretion-powered sources, such as AGNs. Furthermore, hard (e.g. 2-10 keV) X-rays are less affected by dust absorption with respect to other bands. The advent of X-ray imaging surveys with Einstein, ASCA, BeppoSAX, and then XMM-Newton and Chandra have permitted us to improve dramatically our understanding of accretion-powered sources and their cosmic evolution. These surveys provided the evidence of a strong luminosity dependence in the evolution, low luminosity sources (i.e. Seyfert galaxies) peaking at significantly later cosmic times than high luminosity sources (Hasinger 2003; Ueda et al. 2003; La Franca et al. 2005), at variance with the results of the COMBO-17 survey (Wolf et al. 2003), and the redshift of maximum comoving density ranging from $\sim 1$ to $\sim 0.5$. It is obvious that part of the discrepancy is due to the difficulty in selecting type 2 (absorbed) AGNs using optical techniques, but, even restricting our attention to type 1 objects, the discrepancy maintains and there is evidence that this is due to the incompleteness of optical techniques in selecting LLAGNs (Bongiorno et al. 2007).

Selection by variability has enabled us to identify objects of intrinsically low X-ray to optical ratio, which would be otherwise missed by X-ray surveys (Sarajedini et al. 2003, 2006; Trevese et al. 2007). These objects, in addition to their contribution to the LF evolution, provide information on accretion and/or on star-burst activity.

Repeated observations of the same area of sky enable the detection and study of various classes of astronomical objects, such as variable stars, supernovae, planetary systems, fast moving objects and AGNs. The Large Synoptic Survey Telescope ${ }^{1}$ (LSST) foreseen in 2014 (Ivezic 2007) will address most of these issues and will be able to detect AGNs with high completeness to very faint limits (Green et al. 2007). In the mean time, wide field and deep supernova surveys like ESSENCE (Miknaitis et al. 2007), are already providing data that can be analysed for AGN selection. In this paper we report the AGN detection through variability in one of the fields of the Southern inTermediate Redshift ESO Supernova Search (STRESS) (Botticella et al. 2008) and we discuss the properties of the variability-selected objects. The paper is organised as follows. Section 1 describes the data; Sect. 2 describes the method adopted to select the AGN candidates and the resulting sample; Sect. 3 discusses the optical properties of the AGN candidates; Sect. 4 discusses their X-ray properties and Sect. 5 contains a summary of the results. We

1 http://www. lsst.org adopt throughout the cosmology $H=75 \mathrm{~km} \mathrm{~s}^{-1} \mathrm{Mpc}^{-1}, \Omega_{\mathrm{M}}=$ $0.3, \Omega_{\Lambda}=0.7$.

\section{The STRESS supernova search programme}

Our detection of variable objects is based on a new analysis of images from the Southern inTermediate Redshift ESO Supernova Search (STRESS), which is a long term project designed to measure the evolution in cosmic time of the rate of all types of Supernova (SN) events (Cappellaro et al. 2005; Botticella et al. 2008). The supernova search is based on the comparison of images of selected sky fields obtained at different epochs. In general, the temporal sampling of the observations is tuned to the specific goal to be achieved. To ensure that all $\mathrm{SNe}$ are detected within the time that elapsed between the first and the last observation of a given field, the time baseline must be longer than the time for significant luminosity evolution of all SN types, i.e. as long as 3-4 months. For STRESS, 21 fields were initially selected. They are evenly distributed in right ascension, and have been monitored for about 2 years with an average sampling of one observation every three months. A typical observing run was divided in two parts: the search for and follow-up observation of candidates. The search was conducted during two consecutive nights at the ESO/MPI 2.2 m telescope at ESO, La Silla (Chile). The telescope was equipped with the Wide Field Imager (WFI) and a mosaic of $2 \times 4$ CCD detectors of $2048 \times 4096$ pixels that image a sky area of $0.25 \mathrm{deg}^{2}$ with an excellent spatial resolution of $0.238 \mathrm{arcsec} / \mathrm{pixel}$. When possible, the first observing night was dedicated to obtaining deep $V$ band exposures for candidate detection, while in the second night the same fields were observed using a different filter, $B$ or $R$, to collect colour information both for the candidates and the galaxies. Due to a number of technical, meteorological, and scheduling constraints, in many cases it was impossible to maintain this observing strategy. This implies that only in a few cases it was not possible to derive the candidate colour. For this reason, in the following we consider only the $V$ band exposures and derive the candidate colours from another survey, as discussed in Sect. 4. To remove detector cosmetic defects, cosmic rays, satellite tracks and fast moving objects, we acquired for each field three frames dithered by $5-10 \mathrm{arcsec}$, for a total exposure time of $900 \mathrm{~s}$ or in some cases $600 \mathrm{~s}$, as reported in Table 1. For each field, the difference between the image to be searched (target image) and a suitable archive frame (template image) was computed. After accurate astrometric and photometric registrations, the most crucial step in this process was the matching of the point spread function (PSF) of the two images. This was done using the ISIS2.1 package (Alard 2000) that, from comparison of the same sources in the two images, computes a spatially-varying convolution kernel to degrade the image with the best seeing to match the other one. Variable sources leave residuals in the difference image, which were detected and logged into a catalogue using the SExtractor program (Bertin \& Arnouts 1996). After series of checks to remove false detections, residuals of moving objects and variable stars, one is left with a few SN candidates, typically from none to a handful per field. More details on the photometric analysis and the follow up spectroscopic campaign are described in Cappellaro et al. (2005) and Botticella et al. (2008). For the purpose of the present work, we note only that, on the basis of spectra obtained at VLT, about $75 \%$ of the supernova candidates were confirmed and almost all of the remaining $25 \%$ were found to be AGNs.

The approach to candidate selection was designed to avoid as far as possible any selection bias and in particular nuclear 
Table 1. AXAF field observation log.

\begin{tabular}{lccc}
\hline \hline Name of image & $\begin{array}{c}\text { Date } \\
\text { (yyyy-mm-dd) }\end{array}$ & $\begin{array}{c}\text { Exposure time } \\
(\mathrm{s})\end{array}$ & $\begin{array}{c}\text { Seeing } \\
\text { (arcsec) }\end{array}$ \\
\hline AXAF_V_19991109 & $1999-11-09$ & 900 & 1.08 \\
AXAF_V_19991202 & $1999-12-02$ & 900 & 1.12 \\
AXAF_V_19991228 & $1999-12-28$ & 900 & 1.15 \\
AXAF_V_20001116 & $2000-11-16$ & 600 & 0.92 \\
AXAF_V_20001217 & $2000-12-17$ & 600 & 0.93 \\
AXAF_V_20011112 & $2001-11-12$ & 900 & 1.03 \\
AXAF_V_20011118 & $2001-11-18$ & 900 & 0.84 \\
AXAF_V_20011208 & $2001-12-08$ & 900 & 0.95 \\
\hline
\end{tabular}

candidates were not excluded a priori. Given that, the intrusion of AGNs is unavoidable. For the purpose of SNe searches, contamination by variable AGNs was reduced by looking at the long term variability history of the candidates. Sources showing longterm, erratic variability, were excluded from the list of SN candidates. While AGNs represent a contamination of the SNe sample, they are in fact the targets of the present analysis. Thus, we flag as possible SN contaminants those objects that present a "single flare". Moreover, we are only interested in nuclear variation. This suggests the use of aperture photometry instead of image subtraction, and a statistical approach to variability detection, described in the following section. As shown in the next section, the number of AGN candidates exceed $\mathrm{SN}$ candidates by about 44 times in our variable source selection, which is optimised for AGN detection down to $V \sim 24 \mathrm{mag}$, i.e. we expect a contamination of less than $3 \mathrm{SNe}$ candidates, which is of minor concern. In principle, we can select AGN candidates in the entire collection of fields monitored by the STRESS programme. As a first step, we selected the field named AXAF (after the name of the X-ray AXAF satellite subsequently called Chandra), centred on 03:32:23.7 -27:55:52 (J2000) and covering $\sim 0.25 \mathrm{deg}^{2}$.

Table 1 reports the names, dates, and exposure times of the set of 8 images used in the present analysis, acquired at the ESO $2.2 \mathrm{~m}$ telescope with the Wide Field Imager in the AXAF field.

This choice is motivated by the fact that this field overlaps: i) the ESO Imaging Survey (EIS) (Arnouts et al. 2001) containing $B, V, R$, and $I$ photometry and a morphological classification based on the SExtractor code; ii) the COMBO-17 survey (Wolf et al. 2004) containing photometry in 3 broad and 14 narrow bands and providing a classification of objects in galaxies, AGNs and stars on the sole basis of their spectral energy distribution (SED); iii) the Chandra Deep Field South (CDFS) survey (Giacconi et al. 2002; Alexander et al. 2003) that is based on $\mathrm{X}$-ray exposures of $1 \mathrm{Ms}$, which has spectroscopic follow up observations described by Szokoly et al. (2004); iv) the Extended Chandra Deep Field South (ECDFS) survey, consisting of four $16.9 \times 16.9 \mathrm{arcmin}^{2}$ pointings of $250 \mathrm{ks}$ each, flanking the CDFS (Lehmer et al. 2005); v) the GOODS survey, which gathers the data from various catalogues, including the above-mentioned ones, and provides also optical spectra for a sizable fraction of our sample (mostly from Giavalisco et al. 2004).

XMM-Newton observations for a total time of $\sim 500 \mathrm{ks}$ and centred on the CDFS are also available (Streblyanska et al. 2004; Dwelly \& Page 2006), but they cover only a fraction of the AXAF field, at a slightly shallower depth with respect to the $1 \mathrm{Ms}$ Chandra observation.

A $V$-band image of the field is shown in Fig. 1, where the fields of the above surveys are also indicated.

In this way, it is possible to analyse the properties of objects selected by means of variability, by using, in particular, X-ray

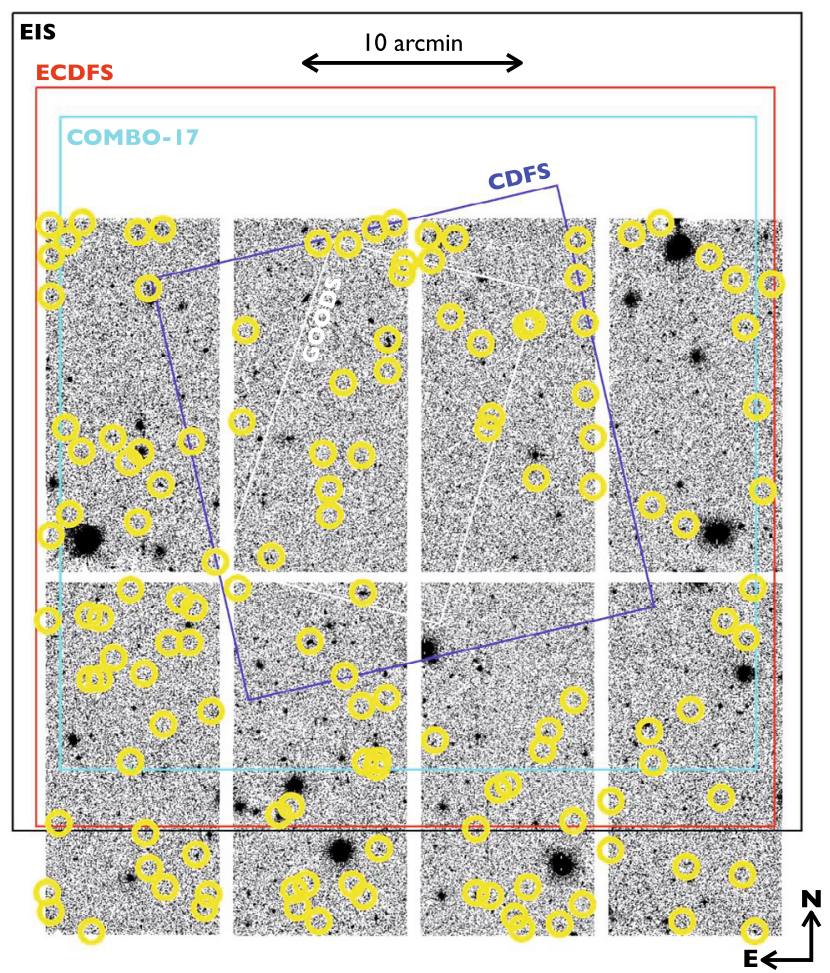

Fig. 1. $V$ band image of the AXAF field taken with the Wide Field Imager at the $2.2 \mathrm{~m}$ ESO telescope. Contours delimit the area of: i) the ESO Imaging Survey (EIS) (Arnouts et al. 2001) (black); ii) the COMBO-17 survey (Wolf et al. 2004) (cyan); iii) the Chandra Deep Field South (CDFS) 1 Ms survey (Giacconi et al. 2002; Alexander et al. 2003) (blue); the Extended Chandra Deep Field South (ECDFS) 250 ks survey (Lehmer et al. 2005) (red); v) the GOODS survey (white) (Giavalisco et al. 2004). Yellow circles represent the variable objects reported in Table 2 .

emission and optical spectra, whenever available; data about optical and image extension also provide valuable information.

\section{Photometry and selection of variable objects}

Objects were detected using SExtractor on each of the 8 images corresponding to different epochs of observation of the AXAF field. The image AXAF_V_20011208, corresponding to the 8th epoch (see Table 1), was selected to be the reference image since it contains the highest number of objects, due to its higher quality, and has the best overlap area with the field of the other images. The corresponding catalogue was then assumed to be the reference catalogue. Aperture photometry was performed for each object on all images at the same $(\alpha, \delta)$ positions, for various apertures.

The $V$ magnitudes, reported in Col. 4 of Table 2, are scaled to $(\mathrm{AB})$ system magnitudes, as taken from the EIS catalogue that we use for colour information. More specifically the zero point of our scale is defined by the condition $\left\langle V-V_{\mathrm{EIS}}\right\rangle=0$, where the brackets indicate the average taken over all sources with the following constraints: i) non variable, i.e. $\sigma^{*} \leq 3$ (see below); ii) $17<V_{\text {EIS }}<21.5 \mathrm{mag}$; iii) point-like, i.e. with SExtractor stellarity index $>0.9$. The reason for the latter condition is that we used fixed aperture magnitudes, while the EIS catalogue reports "total" magnitudes as measured by SExtractor; for diffuse objects, $V-V_{\mathrm{EIS}}$ therefore depends on the extension of the image, becoming another indicator of stellarity. This can be seen in Fig. 2, where stars occupy a "stellar locus" about 


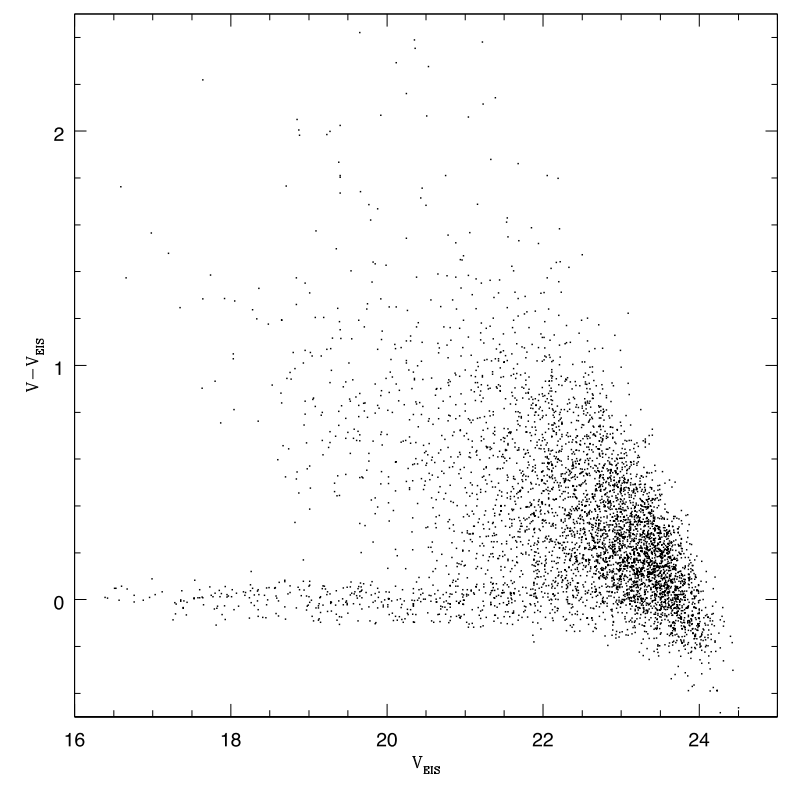

Fig. 2. The difference $V-V_{\text {EIS }}$ between the magnitudes we measured on $2.2 \mathrm{~m}$ WFI images and those reported in the EIS catalogue.

$V-V_{\text {EIS }}=0$, and galaxies are spread over the upper part of the plot. The rms dispersion of the stellar locus provides an indication of the (quadratically) combined photometric uncertainties of EIS magnitudes and our own, which are 0.048, 0.056, and 0.11, for $17<V_{\text {EIS }}<19,19<V_{\text {EIS }}<21.5$, and $21.5<V_{\text {EIS }}<22.5$ respectively. The completeness limit of our catalogue is about $V=24$.

Given any image pair $(i, j)$, we compute the mean square difference $\left\langle\left(m_{i}-m_{j}\right)^{2}\right\rangle \equiv \Sigma_{i, j}^{2}=\Sigma_{i}^{2}+\Sigma_{j}^{2}$ between the magnitudes of all objects at two epochs $(i, j)$, where the angular brackets represent the average computed over all objects, $\Sigma_{i}$ and $\Sigma_{j}$ denote the photometric noise of each image; we neglect the contribution of intrinsically variable objects, since they represent a small fraction of objects in the field. Of course, photometric noise and optimal aperture depend on both observing conditions and apparent magnitude of the object, although in reality the dependence is not extremely strong. For simplicity, we therefore adopted a fixed aperture of 4 pixels ( 0.92 arcsec) radius, for all magnitudes and epochs.

Indicating by $m_{k}^{0}$ the magnitude of the $k$ th object in the reference image, and by $m_{k}^{i}$ the magnitude of the same object in the $i$ th image, and defining $d^{(i, 0)} \equiv\left\langle m_{k}^{i}-m_{k}^{0}\right\rangle$ to be the ensemble average in a given magnitude bin, we computed the relative magnitudes $\mu_{k}^{i}(m) \equiv\left(m_{k}^{i}-m_{k}^{0}\right)-d^{(i, 0)}$, for each object $k$ and each epoch $i$. The amplitude of the magnitude bins was the result of a trade-off between the number of objects in each bin and the necessity of tracing the dependence of $d^{(i, 0)}$ on $m$. The computation of the ensemble average was repeated after a $3-\Sigma_{i, j}$ clipping procedure to minimise the effect on $\mu_{k}^{i}(m)$ of the most deviant points, which probably correspond to intrinsic variations rather than noise fluctuations.

In this way, we derived, for each object in the field, relative light curves, which are independent of the observing conditions (exposure, background light, seeing, CCD amplification). From the light curve of each object $k$, we then compute the mean $\bar{m}_{k}$ and the rms deviation $\sigma_{k}$ :

$\bar{m}_{k}=\frac{1}{N_{\text {epo }}} \sum_{i}^{N_{\text {epo }}} m_{k}^{i}, \quad \sigma_{k}=\left[\frac{1}{N_{\text {epo }}} \sum_{i}^{N_{\text {epo }}}\left(m_{k}-\bar{m}_{k}\right)^{2}\right]^{\frac{1}{2}}$,

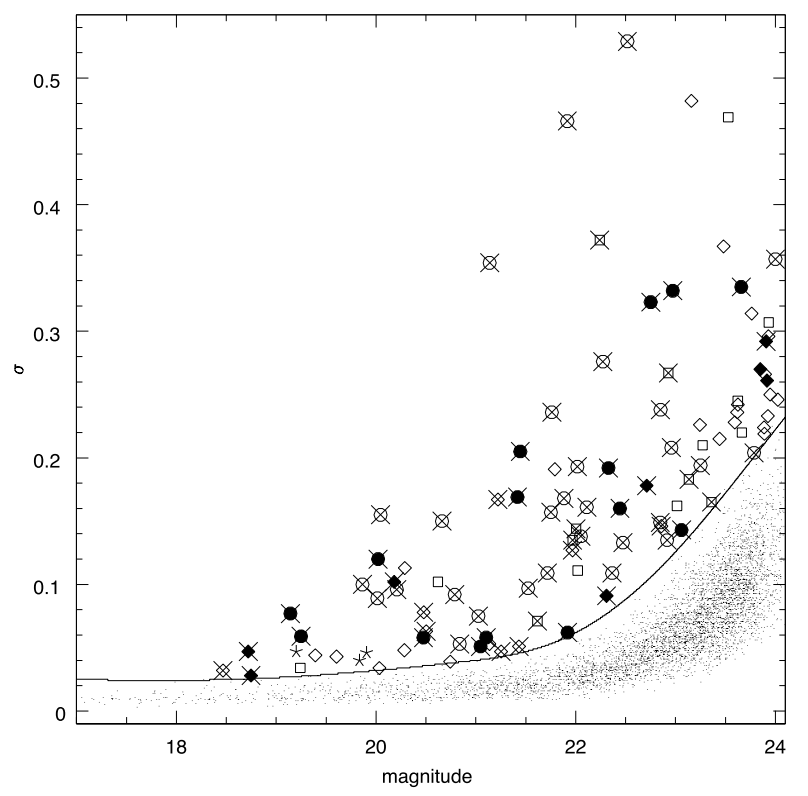

Fig. 3. The rms magnitude variations $\sigma(V)$ as a function of the apparent $V$ magnitude, for all of the objects in the field. Non variable objects are shown as small dots. Symbols representing the classification are not reported below the 3- $\sigma$ variability threshold, which is indicated by the continuous line. Variable objects, above the variability threshold, are indicated as follows. Filled symbols: objects with spectroscopic redshift; empty symbols: objects without spectroscopic redshift; crosses: X-ray detected objects; circles, diamonds, stars: objects classified by COMBO-17 as QSOs, galaxies, stars, respectively; squares: objects outside the COMBO-17 field.

where $N_{\text {epo }}$ is the number of epochs, which in our case is 8 , or smaller in the case of some objects, close to the border, which at some epoch are located outside of the field or are contaminated by hot pixels or other defects. In any case, we decided to consider only objects that had measured magnitudes for at least 5 of the 8 epochs of observation; in this way, we rejected about $2 \%$ of the objects and our final reference catalogue contained 7267 objects brighter than $V=24$. Figure 3 shows $\sigma \equiv \sigma_{k}$ (hereinafter we omit the $k$ subscript for simplicity) versus the $V$ magnitude for all objects in our catalogue. The dependence of both the average $s(V)=\langle\sigma\rangle$ and the rms deviation $\Sigma_{\sigma}(V)=\left\langle[\sigma-s(V)]^{2}\right\rangle^{1 / 2}$ on $V$, where the average is computed in bins of $V$, is clearly evident. The average $s(V)$ represents the rms noise that must be subtracted from $\sigma$ to measure the intrinsic variability. As the number $N_{\text {epo }}$ of observations is increased, $s(V)$ does not vary, but instead it converges to the rms noise. The $\sigma$ of intrinsically variable objects may or may not change, depending on how the intrinsic variability timescale compares with the total duration of the observing campaign and the sampling intervals. The rms deviation $\Sigma_{\sigma}(V)$, instead, decreases as $N_{\text {epo }}^{-1 / 2}$, and thus an increase of the number in observations allows us to decrease the threshold that defines variable objects. To maintain the number of spurious variables produced by the photometric noise at a sufficiently low level, we adopt a $3-\Sigma_{\sigma}$ threshold:

$\sigma \geq\left[s(V)+3 \Sigma_{\sigma}(V)\right]$

The continuous line in Fig. 3 represents the adopted threshold. The rms deviation $\sigma$ is a measure of the average amplitude of magnitude changes, which are due in part to photometric noise 
and in part to intrinsic variability. We can also define for each object a normalized rms deviation (as in Bershady et al. 1998):

$\sigma^{*} \equiv \frac{\sigma-s(V)}{\Sigma_{\sigma}(V)}$

which provides a measure of the significance of the variability. Thus variable objects are defined according to the condition $\sigma^{*} \geq 3$.

From the above discussion, it is clear that this method is not optimal for supernova detection. For instance, if the sampling interval between two observations is comparable or larger than the timescale of SN decay, then $\sigma$ decreases with the number $N_{\text {epo }}$ of observing epochs. For AGNs, instead, $\sigma$ increases on average, at least for delays up to $\sim 50$ years, as shown by the structure function analysis of AGN variability (de Vries et al. 2004, 2005).

Our procedure, once applied to the entire AXAF field, produces a list of 132 candidates reported in Table 2. A spectroscopic follow-up project is necessary in any case, not only to confirm the AGN nature of the candidates and exclude other kinds of variable objects, but also to measure their redshift and assign them to specific classes, such as type 1 or 2 Seyfert galaxies, QSOs, low luminosity AGNs, or star-burst galaxies.

The threshold applied was chosen to be a trade off between the completeness level that we want to achieve and the fraction of spurious candidates (purity or reliability) that we are willing to accept in a follow-up spectroscopic campaign. Purity and completeness are discussed in Sects. 4 and 5 respectively.

\section{Variability-selected AGN candidates}

The colour-colour diagram of Fig. 4 shows all 5138 objects with EIS photometry in the AXAF field, measured at 5 epochs at least. Large symbols represent variable objects reported in Table 2, possessing EIS photometry in the relevant bands: all of them (but one) belong to the ECDFS area (see Fig. 1). Since the fraction of area covered by EIS and not by COMBO-17 is small (see Fig. 1), most objects possess a COMBO-17 classification. More specifically, only 10 objects, represented as open squares, do not possess this classification: 4 of them are detected in X-rays (crosses). Although spectroscopy is required to measure their redshifts and to achieve more detailed classifications, these 4 objects can be considered to be AGNs on the basis of the presence of both variability and X-ray emission (see $\mathrm{Maoz}$ et al. 2005). We evaluate, in addition, their X-ray to optical ratio $(\mathrm{X} / \mathrm{O})$, that is defined on the basis of $R$-band optical flux and 2-8 keV X-ray flux, which is less affected by obscuration than softer X-ray bands. All of these objects have $\log \mathrm{X} / \mathrm{O}>-1$, a value more consistent with AGN activity than starburst galaxies, which typically have $\log \mathrm{X} / \mathrm{O}<-1$ (e.g. Georgakakis et al. 2007). Obviously the opposite is not true, since the host galaxy light may reduce the apparent X/O ratio of a faint AGN, depending on the aperture adopted for optical photometry and the seeing conditions. The other objects represented by open symbols are either QSOs (circles) or galaxies (diamonds), with COMBO-17 classification but without optical spectroscopy. All of the former are also X-ray emitting and, if we consider the high reliability of the COMBO-17 classification for objects dominated by nuclear emission, we can assume that they are bona fide QSOs. Among the variable objects classified as galaxies by COMBO-17 and without spectroscopic redshift (open diamonds), some (7) have also X-ray emission and are likely to be relatively faint AGNs hosted by a galaxy whose light swamps the nuclear radiation. This is indicated by their stellarity index in Fig. 5 where most

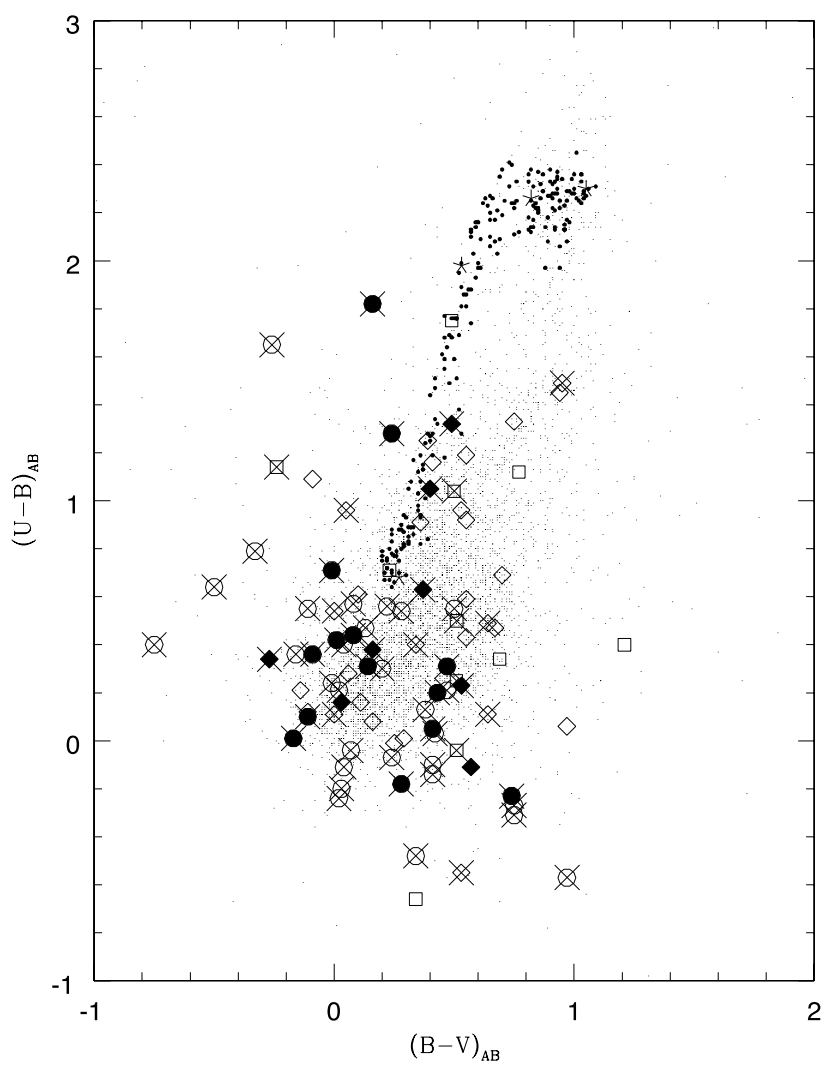

Fig. 4. $(U-B)_{A B}$ versus $(B-V)_{A B}$ colours for all 5138 objects with EIS photometry in the AXAF field and measured at least at 5 epochs. Non-variable galaxies: small dots; non-variable stars: larger dots. Variable objects are indicated as follows: filled symbols, objects with spectroscopic redshift; empty symbols, objects without spectroscopic redshift; crosses, X-ray detected objects; circles, diamonds, stars: objects classified by COMBO-17 as QSOs, galaxies, and stars, respectively; squares, objects outside the COMBO-17 field.

of them (6/7, open diamonds with crosses) are non stellar. The same can be true for objects not detected in X-rays, although, in this case, the nuclear component must be even fainter. Their variability suggests that they are AGN, which, however, requires spectroscopic confirmation. If the redshift is known, an X-ray luminosity $L_{X}(2-8 \mathrm{keV})>10^{42} \mathrm{erg} \mathrm{s}^{-1}$ can be assumed as a more direct indication of the AGN character. All of the spectroscopically confirmed QSOs (14 filled circles) have X-ray emission. Of the galaxies with known redshift (8 filled diamonds), 4 have spectra with broad emission lines, are also detected in X-rays, and are consistent with AGN activity. To evaluate the purity of the sample, i.e. the fraction of AGNs in the variability selected sample, we consider the 104 variable objects in the field covered by X-ray data, 4 of which are variable stars (with a variability in the range $0.03-0.05 \mathrm{mag} \mathrm{rms}$ ). Among these 104 variable objects, we consider bona fide AGNs to be: 44 COMBO-17 QSOs with X-ray emission, 4 COMBO-17 galaxies with broad emission lines, 8 COMBO-17 galaxies without spectrum but with $\log \mathrm{X} / \mathrm{O}>-1$, and 7 objects without COMBO-17 classification but with $\log \mathrm{X} / \mathrm{O}>-1$. This corresponds to a purity of at least $60 \%(63 / 104)$. We stress that this is a lower limit, since most of the remaining variable objects could also hide some, possibly low luminosity, AGN component that future spectroscopy could reveal.

In Fig. 5, the normalised rms variability $\sigma^{*}$ is reported versus the SExtractor stellarity index that ranges from 0 


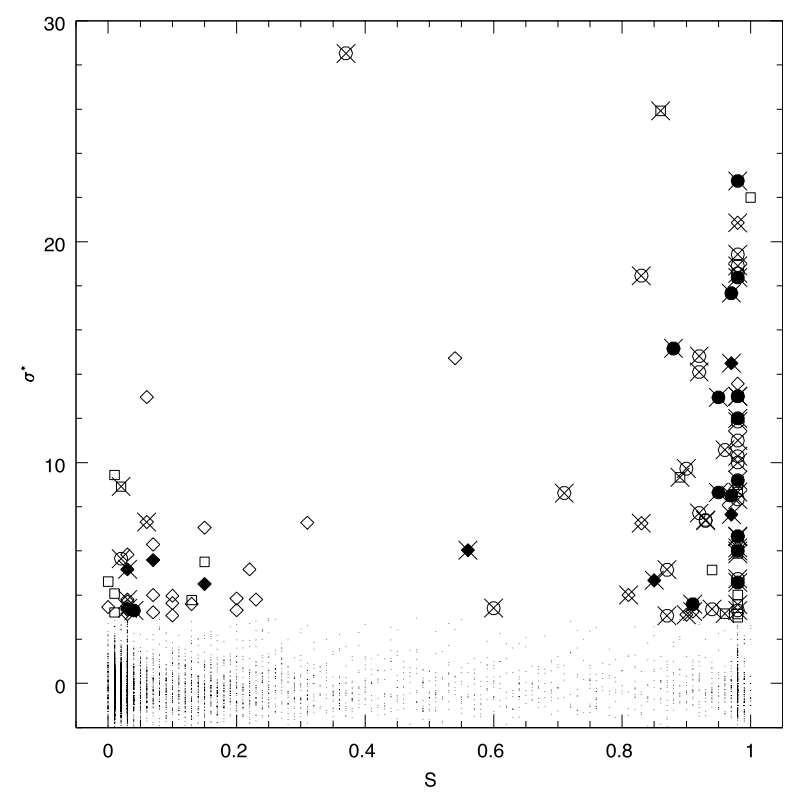

Fig. 5. Normalised rms variability $\sigma^{*}$ versus the SExtractor stellarity index $S$. Symbols as in Figs. 3 and 4.

(extended objects) to 1 (pointlike objects). Small dots are nonvariable sources $\left(\sigma^{*} \leq 3\right)$. Most QSOs, either spectroscopically confirmed or not, tend to have high values of stellarity index, but sometimes between 0.6 and 0.8 and not necessarily very close to 1.0 . This indicates that often the "fuzz" due to the host galaxy is detected by the stellarity index. For two of the COMBO-17 QSOs, one of which is spectroscopically confirmed, the stellarity index is however typical of that of a galaxy. This circumstance implies that further investigation is necessary. One possible explanation is related to variability: for example, the nucleus could have been fainter at the epoch of morphological classification and brighter at the epoch of COMBO-17 SED classification. The same is true for objects classified as COMBO17 galaxies that have a stellarity index close to 1 . It appears that all 4 galaxies with optical spectroscopy (filled diamonds) and a stellarity index greater than 0.5 are X-ray emitting and spectroscopically confirmed AGNs.

\subsection{Comparison with an HST variability survey}

A variability survey with Hubble Space Telescope (HST) Advanced Camera for Surveys (ACS) was undertaken by Klesman \& Sarajedini (2007) in the GOODS-South field, which is contained in our AXAF field. The variability of a composite sample, consisting of 22 mid-IR power-law sources from Alonso-Herrero et al. (2006) and 104 X-ray sources from Alexander et al. (2003), was analysed on the basis of ACS exposures at 5 different epochs separated by 45 day intervals. The ACS analysis was motivated by the special interest of detecting and studying LLAGNs. With the high angular resolution of HST, it is possible to perform photometry using an extremely small aperture: $0.075^{\prime \prime}$ radius in this case. This reduces the diluting effect of the host galaxy light on the nuclear variability, allowing for its detection down to a nuclear magnitude $M_{\mathrm{B}} \sim-15$, as discussed by Sarajedini et al. (2003). Klesman \& Sarajedini (2007) defined a variability measure by the standard deviation $\sigma$ of the light curve, as in the present work, and defined the error in the variability measure $\sigma$ as error $_{\sigma}=\sqrt{\Sigma\left(\text { error }_{\text {mag }}\right)^{2} / N}$,

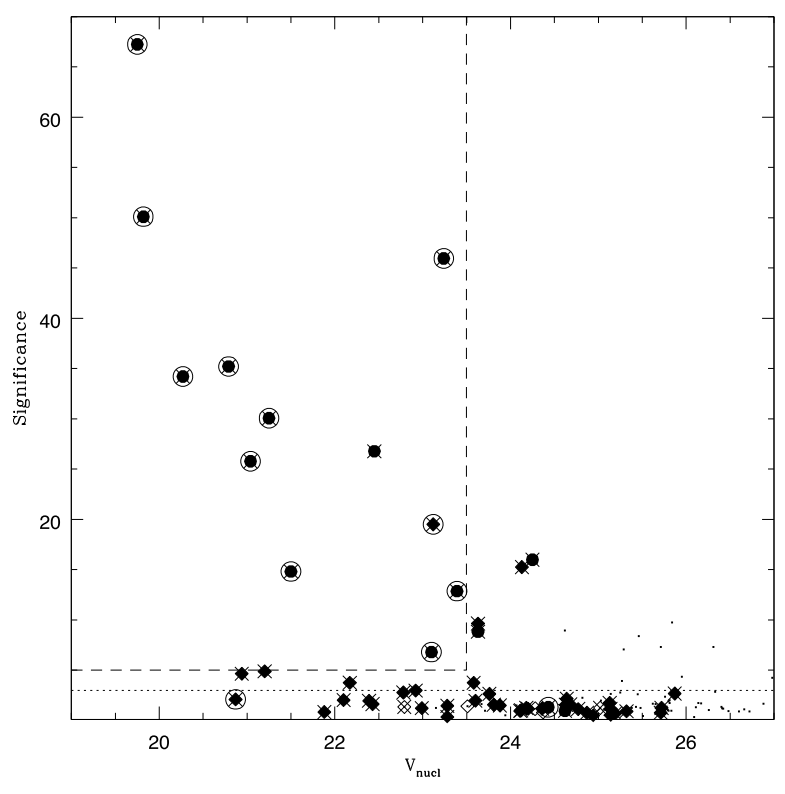

Fig. 6. Significance versus the nuclear magnitude $V_{\text {Nucl }}$ from Klesman \& Sarajedini (2007). Small dots: objects not in our survey; large symbols: objects which appear in the present survey; filled symbols: objects with spectroscopy; open symbols: objects without spectroscopy; diamonds: galaxies from COMBO-17; circles: COMBO-17 QSOs; crosses: X-ray sources; large open circles: variable objects from our survey. Two of the latter are not variable according to Klesman \& Sarajedini (2007) (see text). The horizontal dotted line is the variability threshold of Klesman \& Sarajedini (2007). Within the area delimited by the dashed lines, all objects but one are variable according to both surveys.

where $N$ is the number of epochs in which the object was observed and error $_{\text {mag }}$ is the formal photometric error in the magnitude at each epoch. Although not identical, the definition of error $_{\text {mag }}$ is almost equivalent to our empirical estimate $\Sigma_{\sigma}$ based on the magnitude spread of non-variable objects in each magnitude bin. The significance of variability is then defined to be: significance $=\sigma /$ error $_{\sigma}$. Thus, under the assumption that error $_{\sigma}$ and our $\Sigma_{\sigma}$ are equal to each other (see Eq. (3)), the relation Significance $=\sqrt{\left(\sigma^{*}\right)^{2}+1}$ would hold. We note, however, that the level of noise is not the same in our WFI observations and the ACS observations of Klesman \& Sarajedini (2007).

We compare our data with ACS variability observations, for all objects in common. For these objects, Fig. 6 displays the Significance versus the nuclear magnitude $V_{\text {nucl }}$ taken from Table 1 of Klesman \& Sarajedini (2007).

Objects that appear variable in our survey, i.e. for which $\sigma^{*} \geq 3$, are marked by large open circles. For $V_{\text {nucl }}<23.5$, $11 / 15(73 \%)$ objects that are variable according to Klesman \& Sarajedini (2007), i.e. with Significance $>3$, are variable in our survey. This fraction increases to $11 / 12(92 \%)$, if we consider only objects with Significance $>5$.

Both limits depend on photometric noise. A nuclear magnitude $V_{\text {nucl }}=23.5$ corresponds, on average, to a value of about 23 in our aperture magnitude scale, where the 3- $\sigma$ threshold becomes of the order of 0.1 mag (see Fig. 3). Moreover, the lower angular resolution of ground-based observations tends to dilute nuclear variations and increase the flux-variability threshold, for a given significance. In Fig. 6, there are two objects that appear variable in our survey but lie below the Significance threshold defined by Klesman \& Sarajedini (2007), which is given by the dotted line in Fig. 6. One of these objects is an X-ray detected and spectroscopically confirmed QSO with $V_{\text {nucl }}=24.43$ 


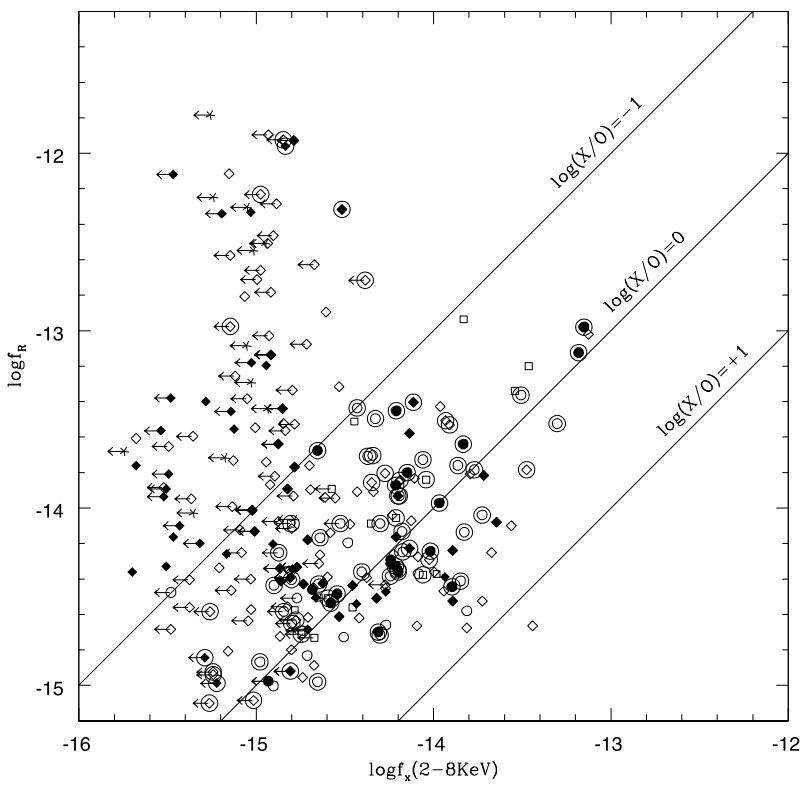

Fig. 7. $\log f_{R}$ versus $\log f_{\mathrm{X}}(2-8 \mathrm{keV})$ for all the objects with measured $\mathrm{X}$-ray flux or with estimated upper limit. Fluxes are taken from Lehmer et al. (2005) when available or from Alexander et al. (2003) or Giacconi et al. (2002) otherwise. Symbols as in Fig. 3. Variable objects are marked by large open circles. Arrows indicate available 3- $\sigma$ upper limits from the same surveys, or from our estimates, for objects undetected in the $(2-8 \mathrm{keV})$ band.

(ID 115 in Table 2). The second (ID 5) is a galaxy with an extranuclear Ultra-Luminous X-ray source (ULX) (Hornschemeier et al. 2004; Lehmer et al. 2006), which could produce extranuclear variability, missed by the small-aperture nuclear photometry of Klesman \& Sarajedini (2007). In any case, the WFI and ACS observations were performed in different periods, and for a total sampling time of 0.5 years in the case of Klesman \& Sarajedini (2007) and, in our case, 2 years. It is, therefore, unsurprising that a few objects are detected as variable in one survey and not in the other.

\section{2. $X$-ray properties}

The X-ray emission is a specific signature of AGNs. The CDFS $1 \mathrm{Ms}$ (Giacconi et al. 2002) is one of the deepest X-ray exposures available in the sky. Additional $1 \mathrm{Ms}$ observations of the CDFS have been performed recently ${ }^{2}$ and a further analysis of the field, including these data and forthcoming new optical spectra, is in progress (Boutsia et al. 2008a). The AXAF field is therefore ideal for a combined study of the X-ray and optical properties of AGNs. In the following, we define $\mathrm{X} / \mathrm{O}$ to be the ratio of the observed fluxes in the $R$ optical band to the fluxes in the $2-8 \mathrm{keV}$ $\mathrm{X}$-ray band. We neglect the $k$-correction that, however, acts in the same sense for both the optical and the X-ray band. Therefore we neglect a difference in $k$-corrections, which is relatively small with respect to the intrinsic spread of the $\mathrm{X} / \mathrm{O}$ values.

Due to the very low limiting flux, of about $3 \times$ $10^{-16} \mathrm{erg} \mathrm{cm}^{-2} \mathrm{~s}^{-1}$ in the $2-8 \mathrm{keV}$ band, the distribution of the optical versus X-ray fluxes, shown in Fig. 7, differs from previous studies with brighter $\mathrm{X}$-ray limits. Below $\sim 5 \times$ $10^{-15} \mathrm{erg} \mathrm{cm}^{-2} \mathrm{~s}^{-1}$, a population of very low X/O objects appears. This is due to the $\mathrm{X}$-ray emission of normal galaxies. Note

2 http://cxc.harvard.edu/cda/whatsnew.html\# $\operatorname{cdfs} 2000-2007$

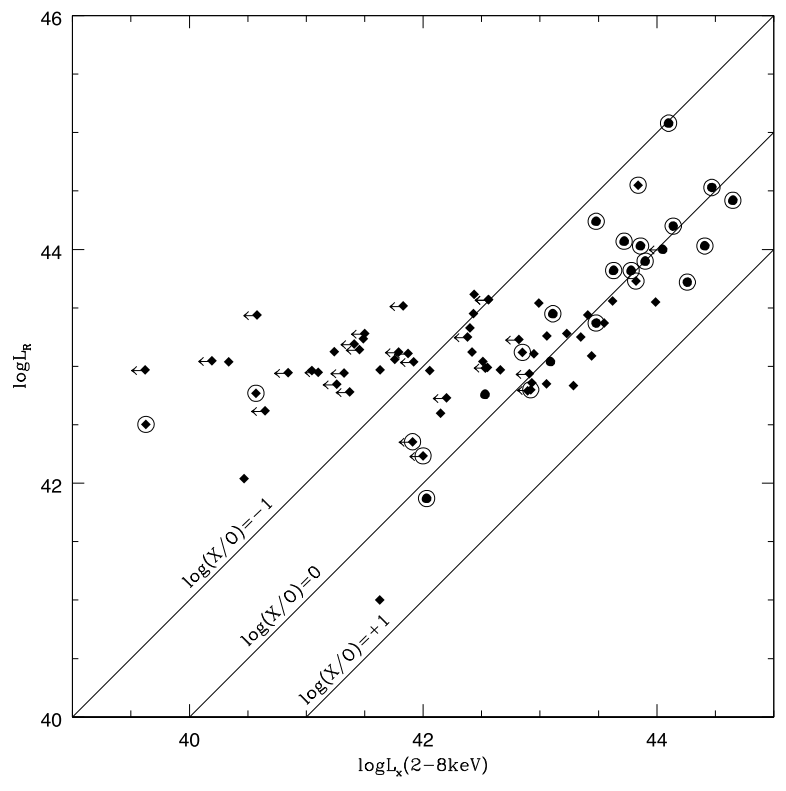

Fig. 8. $\log L_{R}$ versus $\log L_{\mathrm{X}}(2-8 \mathrm{keV})$ for the objects with known redshift. Symbols as in Fig. 7.

that 12 objects, classified as COMBO-17 stars, are in the same $\mathrm{X}$-ray flux range and have $-4 \lesssim \log (\mathrm{X} / \mathrm{O}) \lesssim-1$.

The majority of variable objects (large circles) are concentrated in the $-1<\log (\mathrm{X} / \mathrm{O})<+1$ stripe in Fig. 7, which correspond to typical AGNs. However, six variable objects have lower X/O values, all of which have COMBO-17 SEDs of normal galaxies (diamonds). Two objects (ID 4 and 5 in Table 2) are detected in X-rays, have low $\log (\mathrm{X} / \mathrm{O})(-2.2$ and -2.9$)$, and correspond to extranuclear X-ray sources (Hornschemeier et al. 2004; Lehmer et al. 2006). For all the X-ray undetected variable objects, we have calculated 3- $\sigma$ upper limits to the X-ray flux, from the $1 \mathrm{Ms}$ Chandra images, if not already available from Lehmer et al. (2005) or Alexander et al. (2003). All the upper limits, for variable and non-variable objects, are indicated by leftward arrows in Fig. 7. The lowest upper limit to $\log (\mathrm{X} / \mathrm{O})$ for variable objects is equal to approximately -3 and corresponds to the object ID 3.

For objects of known redshift, we consider the optical versus X-ray luminosity distribution, shown in Fig. 8. There are 18 objects that are classified as COMBO-17 QSOs, and all are broad line AGNs, according to Szokoly et al. (2004), and most of them (15/18) are variable. Including COMBO-17 galaxies, there are 23 variable objects, the majority of which (19/23) have a measured X-ray luminosity $L_{X}(2-8 \mathrm{keV})>10^{42} \mathrm{erg} \mathrm{s}^{-1}$, a value typically used to distinguish between AGN and normal galactic emission. Most of the variable objects occupy the stripe $-1<\log (\mathrm{X} / \mathrm{O})<+1$. We note that 4 of these $(21 \%)$ were classified as COMBO-17 galaxies but show an emission line spectrum of broad line AGNs (ID 23, 75, 83, 125). Two (ID 75, 125) have relatively low SExtractor stellarity index, and would therefore not be detected by usual colour technique, probably due to the relevance of the host galaxy light. Of the 4 variable objects below $10^{42} \mathrm{erg} \mathrm{s}^{-1}, 2$ are the above mentioned ULXs, and the other 2 have upper limits consistent with normal AGNs.

\section{Discussion and summary}

As discussed in the Introduction, the discovery of AGN variability predates the concept of AGN, and various samples have been 
created on the basis of this selection technique. However the amplitude of variability depends on the timescale, type of object, and its luminosity. The properties of the selected sample, such as completeness, purity, and the type of detected objects, depend on the sampling interval, number of observing epochs, total duration of the observing campaign, observing band, and photometric accuracy. The principal aim of the present work was to analyse the properties of AGN candidates selected on the basis of their variability from a photometric campaign, whose sampling rate and duration were developed especially for the detection of supernovae. Large SNe surveys are (and will be) conducted in view of their crucial importance primarily in constraining the dark matter/energy of cosmological models, but also the evolution in cosmic time of the galaxy population. The creation of large statistical samples of AGNs as a by-product of SNe surveys may add scientific value to these surveys without increasing their cost in terms of observing time or special requirements. To demonstrate this, we have selected from the STRESS survey a field that was observed 8 times over 2 years, and was studied in detail by various other surveys, including the $1 \mathrm{Ms}$ X-ray survey of the CDFS. As discussed in Sect. 4, our 3- $\sigma$ variability selection produces a sample of good purity, for which the percentage of "true" AGNs is about $60 \%$ of the total number of AGN candidates. This is a lower limit since a fraction of unconfirmed candidates may also be AGNs. The completeness of the present sample, estimated considering only objects with known spectra and $\mathrm{X}$-ray luminosity $L_{\mathrm{X}}(2-8) \mathrm{keV} \geq 10^{42} \mathrm{erg} \mathrm{s}^{-1}$, is $\sim 44 \%$ (19/43, see Fig. 8), which may increase with further spectroscopic observations (Boutsia et al. 2008a). A relatively high incompleteness suggests caution in basing evolutionary studies on a single selection technique. Still, variability selection may complement the most common surveys based on optical colours or X-ray emission, which suffer different biases.

We stress that by increasing the photometric accuracy and sampling rate, variability surveys can reach far higher completeness. For instance, Sesar et al. (2007) derived 90\% completeness with a threshold of 0.03 mag rms for objects brighter than $g=19.5$, maintaining the contamination of the sample at an acceptable level. A survey to be conducted by the forthcoming LSST may reach a completeness level of $100 \%$ at a limiting magnitude $i<24$, with 12 exposures distributed over a year, according to numerical simulations (Green et al. 2007) based on the extrapolation of statistical properties of AGN variability, as quantified by Vanden Berk et al. (2004) from a variability study conducted on a sample of 25000 QSOs observed by the SDSS. We notice also that, although variability is one of the principal characteristics of active galactic nuclei, its nature is still poorly understood. Independent models have been suggested, including supernova explosions, microlensing, and accretion disk instabilities (Aretxaga et al. 1997; Hawkins 1993; Kawaguchi et al. 1998; Trevese \& Vagnetti 2002). A comparison of these models is discussed in Hawkins (2007). Multi-band variability studies of statistical AGN samples can further constrain the physical origin of luminosity changes.

In general, objects that appear to be galaxies, due to their extended images or their SEDs, are lost by the colour technique (see Fig. 4). This happens in the case of LLAGNs, swamped by the host galaxy light. To reach the lowest possible luminosity limit, variability must be studied using the highest possible spatial resolution, as achieved for HST images both in the HST-N and in the Groth strip fields by Sarajedini et al. (2003, 2006), to reduce the dilution of the nuclear light. Atmospheric seeing obviously prevents us from reaching the same luminosity limits with ground-based observations. The comparison of the results for our different data sets, based on $\sim 15$ min exposures at the ESO $2.2 \mathrm{~m}$ telescope, and the variability study of Klesman \& Sarajedini (2007), based on ACS/HST images of X-ray selected AGNs, indicates that we detect variability at a confidence level of 3- $\sigma$, down to $V_{\text {nucl }} \sim 23.5$, for $70 \%$ of the objects detected as variable sources at a confidence level of 3- $\sigma$ by these authors. Among these objects, the faintest that we observe to be 3- $\sigma$ variable has $V_{\text {nucl }}=24.43$, while the analysis of Klesman \& Sarajedini (2007) detects variability down to $V_{\text {nucl }} \sim 27$. However the area covered by a single field of the STRESS survey is about $900 \mathrm{arcmin}^{2}$. The STRESS project covers 16 fields, thus can provide about 16 times the 130 variable candidates of the present study, i.e. 2000, of which about 1200 are expected to be bona fide AGNs on the basis of the high purity $(60 \%)$ of our sample. This number would allow us to assess statistically the selection effects by comparison with colour-selected or X-ray-selected samples. In a previous paper (Trevese et al. 2008), we analysed the X-ray and optical properties of variability-selected objects in SA 57 and found that several "variable galaxies" are NELGs, for which it was impossible to assess the starburst or LINER nature due to insufficient wavelength coverage. These variable objects are in all cases interesting. If they are LINERs, their variability would imply that they are AGNs, following the argument of Maoz et al. (2005), that the observed variability cannot be accounted for by the luminosity variations of B stars. We note that this is a fortiori true if we consider observations at longer wavelengths than those in the UV discussed by Maoz et al. (2005), despite the shallow decrease in nuclear variability with redshift (Giallongo et al. 1991; Trevese et al. 2001; Vanden Berk et al. 2004). If our objects are instead starburst galaxies, the origin of their variability would present an interesting problem, as in the case of the objects ID 4 and ID 5 in Table 2, which show evidence of extranuclear emission possibly related to ULXs (Hornschemeier et al. 2004; Lehmer et al. 2006).

We note that, from data collected so far by the ESSENCE supernova survey, in 32 fields of a total area of $8 \mathrm{deg}^{2}$, it should be possible to select about 4000 candidates on the basis of variability (Boutsia et al. 2008b); this will increase the number of variable NELGs available and improve our knowledge of the relation between the starburst and AGN phenomenon, providing a development of the synergic SN/AGN survey (cf. conference proceedings about this subject, Bono et al. 2003; Trevese \& Vagnetti 2004).

Finally, the $520 \mathrm{arcmin}^{2}$ field of the Large Binocular Camera (Giallongo et al. 2008) for both red and blue arm of the Large Binocular Telescope, which has at present the widest field of view available for an $8 \mathrm{~m}$ class telescope, will provide an unprecedented opportunity for deep synergic SN/AGN surveys.

Acknowledgements. We acknowledge B. Leibundgut and R. Nesci for discussions and M. T. Botticella for providing details on the STRESS images and help in their handling. This work was partly supported by MIUR under grant PRIN 2006/025203. K.B. acknowledges support by ESO studentship program. This research has made use of the NASA/IPAC Extragalactic Database (NED) which is operated by the Jet Propulsion Laboratory, California Institute of Technology, under contract with the National Aeronautics and Space Administration.

\section{References}

Alexander, D. M., Bauer, F. E., Brandt, W. N., et al. 2005, AJ, 126, 539 Alonso-Herrero, A., Pe'rez-Gonza'lez, P. G., Alexander, D. M., et al. 2006, ApJ, 640,167

Anderson, S. F., Fan, X., Richards, G. T., et al. 2001, AJ, 122, 503

Aretxaga, I., Cid Fernades, R., \& Terlevich, R. 1997, MNRAS, 286, 271 
Arnouts, S., Vandame, B., Benoist, C., et al. 2001, A\&A, 379, 740

Bertin, E., \& Arnouts, S. 1996, A\&AS, 117, 393

Boyle, B. J., Shanks, T., Croom, S. M., et al. 2000, MNRAS, 317, 1014

Bershady, M. A., Trevese, D., \& Kron, R. G. 1998, ApJ, 496, 103

Bongiorno, A., Zamorani, G., Gavignaud, I., et al. 2007, A\&A, 472, 443

Botticella, M. T., Riello, M., Cappellaro, E., et al. 2008, A\&A, 479, 49

Bono, G., Castellani, M., \& Trevese, D. 2003, Variability with Wide-Field Imagers, Mem. SAIt, 74, 851

Boutsia, et al. 2008a, in preparation

Boutsia, et al. 2008b, in preparation

Cappellaro, E., Riello, M., Altavilla, G., et al. 2005, A\&A, 430, 83

Cristiani, S., Vio, R., \& Andreani, P. 1990, AJ, 100, 56

Cristiani, S., Trentini, S., La Franca, F., et al. 1996, A\&A, 306, 395

Croom, S. M., Smith, R. J., Boyle, B. J., et al. 2001, MNRAS, 322, L29

de Vries, W. H., Becker, R. H., \& White, R. L. 2003, AJ, 126, 1217

de Vries, W. H., Becker, R. H., White, R. L., \& Loomis, C. 2005, AJ, 129, 615

Dwelly, T., \& Page, M. J. 2006, MNRAS, 372, 1755

Fan, X., Strauss, M. A., Schneider, D. P., et al. 2001, AJ, 121, 54

Geha, M., Alcock, C., Allsman, R. A., et al. 2003, ApJ, 125, 1

Georgakakis, A., Rowan-Robinson, M., Babbedge, T. S. R., \& Georgantopoulos, I. 2007, MNRAS, 377, 203

Giacconi, R., Zirm, A., \& Wang, J. 2002, ApJS, 139, 369

Giallongo, E., Trevese, D., \& Vagnetti, F. 1991, ApJ, 377, 345

Giallongo, E., Ragazzoni, R., Grazian, A., et al. 2008, A\&A, 482, 349

Giavalisco, M., Ferguson, H. C., \& Koekemoer, A. M. 2004, ApJ, 600, 93

Grazian, A., Fontana, A., de Santis, C., et al. 2006, A\&A, 449, 951

Green, R. F., Brandt, W. N., Vanden Berk, D. E., Schneider, D. P., \& Osmer, P. S 2007, ASP Conf. Ser., 373, 707

Hasinger, G. 2003, AIP conf., 666, 227

Hawkins, M. R. S. 1983, MNRAS, 202, 571

Hawkins, M. R. S. 1993, Nature, 366, 242

Hawkins, M. R. S. 2007, A\&A, 462, 581

Hook, I. M., McMahon, R. G., Boyle, B. J., \& Irwin, M. J. 1994, MNRAS, 268, 305

Hornschemeier, A. E., Alexander, D. M., Bauer, F. E., et al. 2004, ApJ, 600, L47

Ivezic, Z. 2007, Bull. Am. Astron. Soc., 38, 174

Jiang, L., Fan, X., Ivezic, Z., et al. 2007, ApJ, 656, 680

Kawaguchi, T., Mineshige, S., Umemura, M., \& Turner, E. L. 1998, ApJ, 504, 671

Kellermann, K. I., Sramek, R., \& Schmidt, M. 1989, AJ, 98, 1195
Klesman, A., \& Sarajedini, V. 2007, ApJ, 665, 225

Koo, D. C., \& Kron, R. G. 1988, ApJ, 325, 92

Koo, D. C., Kron, R. G., \& Cudworth, K. M. 1986, PASP, 98, 285

La Franca, F., Fiore, F., Comastri, A., et al. 2005, ApJ, 635, 864

Lehmer, B. D., Brandt, W. N., Alexander, D. M., et al. 2005, ApJS, 161, 21

Lehmer, B. D., Brandt, W. N., Hornschemeier, A. E., et al. 2006, AJ, 131, 2394

Matthews, T. A., \& Sandage, A. R. 1963, ApJ, 138, 30

Maoz, D., Nagar, N. M., Falcke, H., \& Wilson, A. S. 2005, ApJ, 625, 699

Miknaitis, G. 2007, ApJ, 666, 674

Ravikumar, C. D., Puech, M., Flores, H., et al. 2007, A\&A, 465, 1099

Richards, G. T., Strauss, M. A., Fan, X., et al. 2006, AJ, 131, 2766

Sarajedini, V. L., Gilliland, R. L., \& Kasm, C. 2003, ApJ, 599, 173

Sarajedini, V. L., Koo, D. C., Phillips, A. C., et al. 2006, ApJS, 166, 69

Schmidt, M. 1968, ApJ, 151, 393

Schmidt, M. 1970, ApJ, 16, 371

Schmidt, M., Schneider, D. P., \& Gunn, J. E. 1995, AJ, 110, 68

Sesar, B., Ivezic, Z., Lupton, R. H., et al. 2007, AJ, 134, 2236

Streblyanska, A., Bergeron, J., Brunner, H., et al. 2004, Nuc. Phys. B Proc. Suppl., 132, 232

Szokoly, G. P., Bergeron, J., Hasinger, G., et al. 2004, ApJS, 155, 271

Trevese, D., \& Vagnetti, F. 2002, ApJ, 564, 630

Trevese, D., \& Vagnetti, F. 2004, Sinergies in Wide-Field Observations, Baltic Astro., 13, 611

Trevese, D., Pittella, G., Kron, R. G., Koo, D. C., \& Bershady, M. A. 1989, AJ, 98, 108

Trevese, D., Kron, R. G., Majewski, S. R., Bershady, M. A., \& Koo, D. C. 1994, ApJ, 433, 494

Trevese, D., Kron, R. G., \& Bunone, A. 2001, ApJ, 551, 103

Trevese, D., Vagnetti, F., Puccetti, S., et al. 2007, A\&A, 469, 1211

Trevese, D., Vagnetti, F., Zitelli, V., Boutsia, K., \& Stirpe, G. M. 2008, A\&A, 477, 473

Ueda, Y., Akijama, M., Ohta, K., \& Miyaji, T. 2003, ApJ, 598, 886

Usher, P. D. 1978, ApJ, 222, 40

van den Berg, S., Herbst, E., \& Pritchet, C. 1973, AJ, 78, 375

Vanden Berk, D. E., Wilhite, B. C., Kron, R. G., et al. 2004, ApJ, 601, 692

Véron, P., \& Hawkins, M. R. S. 1995, A\&A, 296, 665

Warren, S. J., Hewett, P. C., \& Osmer, P. S. 1994, ApJ, 421, 412

Wolf, C., Wisotzki, L., Borch, A., et al. 2003, A\&A, 408, 499

Wolf, C., Meisenheimer, K., Kleinheinrich, M., et al. 2004, A\&A, 421, 913 
D. Trevese et al.: Variability-selected AGNs, Online Material p 1

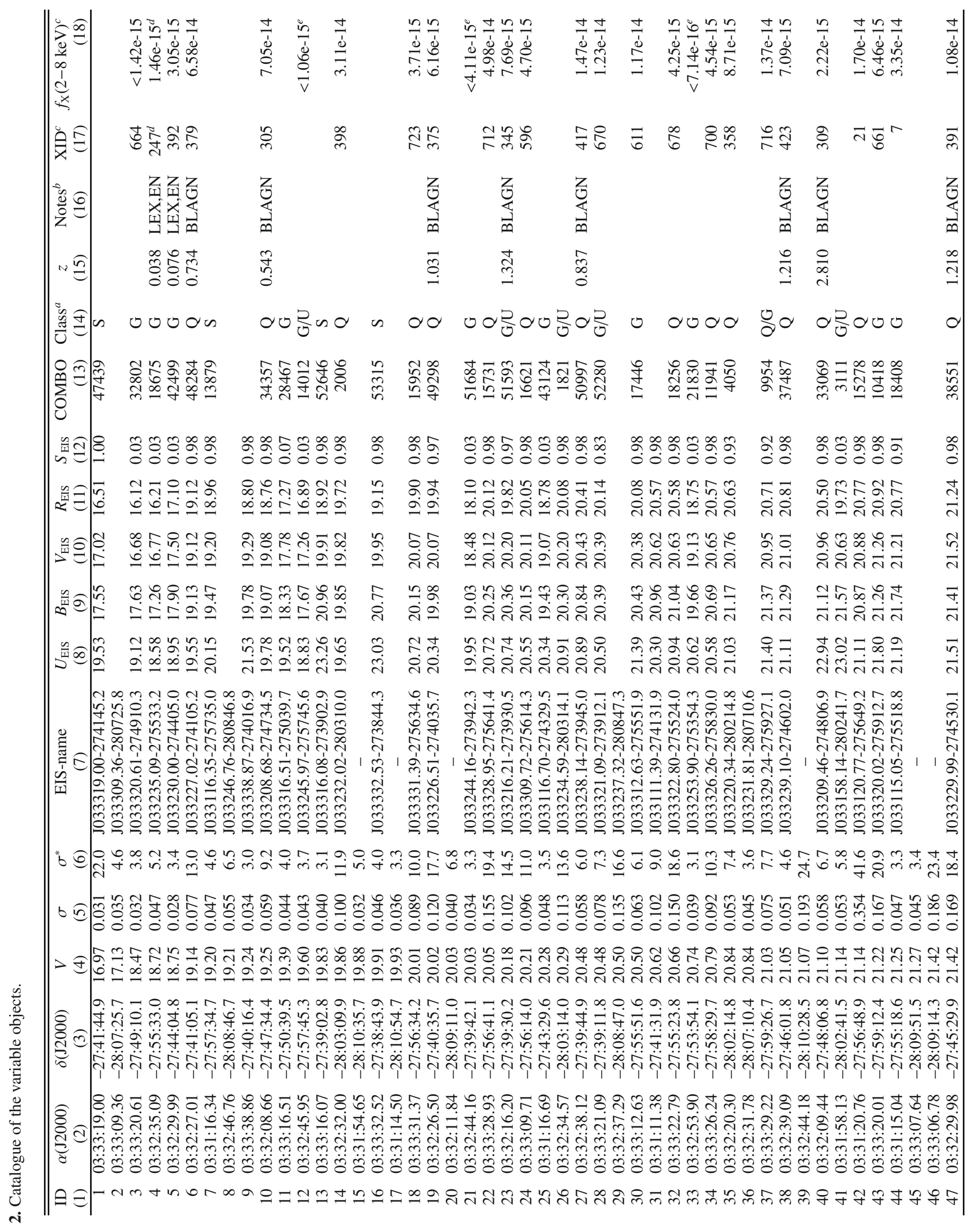


D. Trevese et al.: Variability-selected AGNs, Online Material p 2

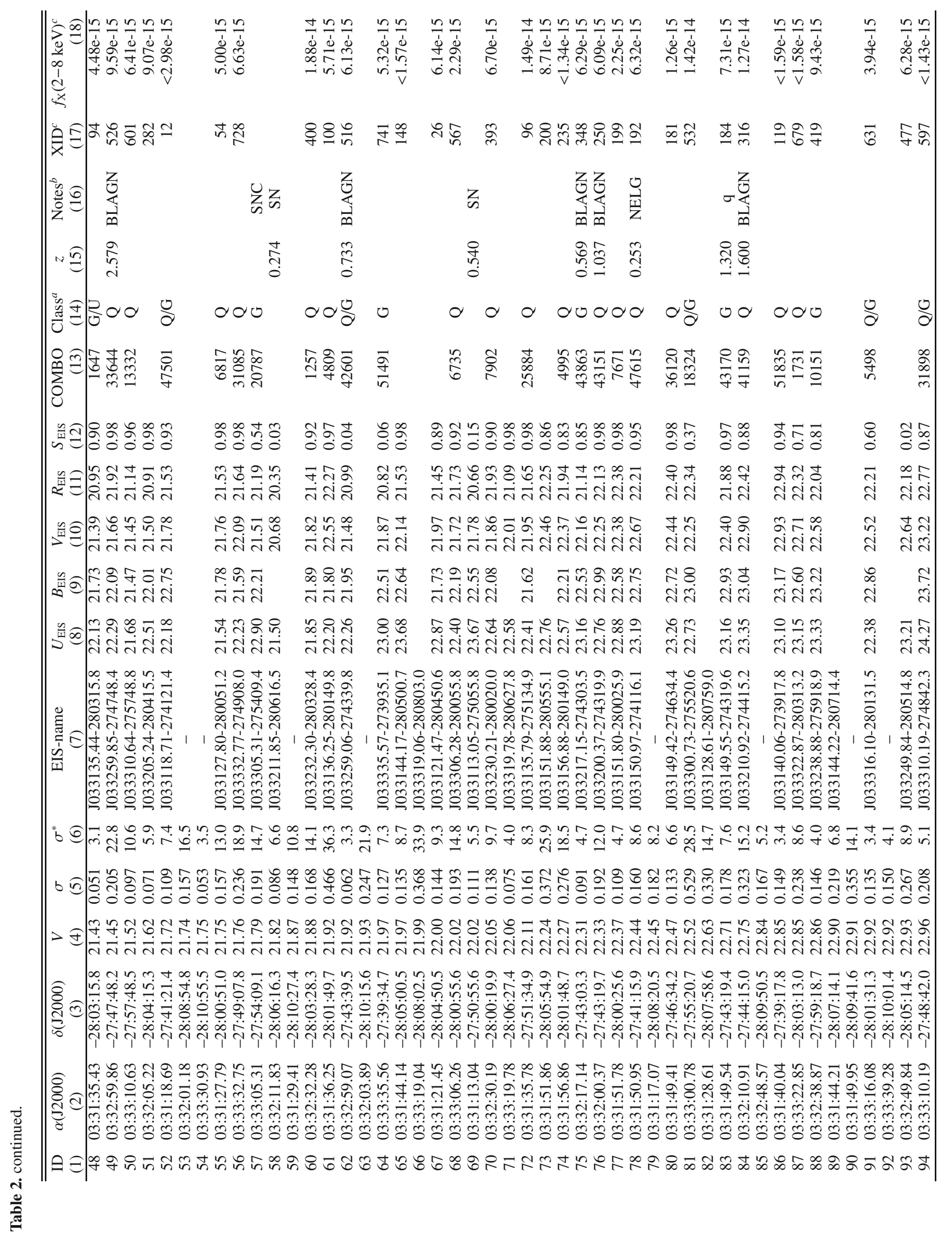


D. Trevese et al.: Variability-selected AGNs, Online Material p 3

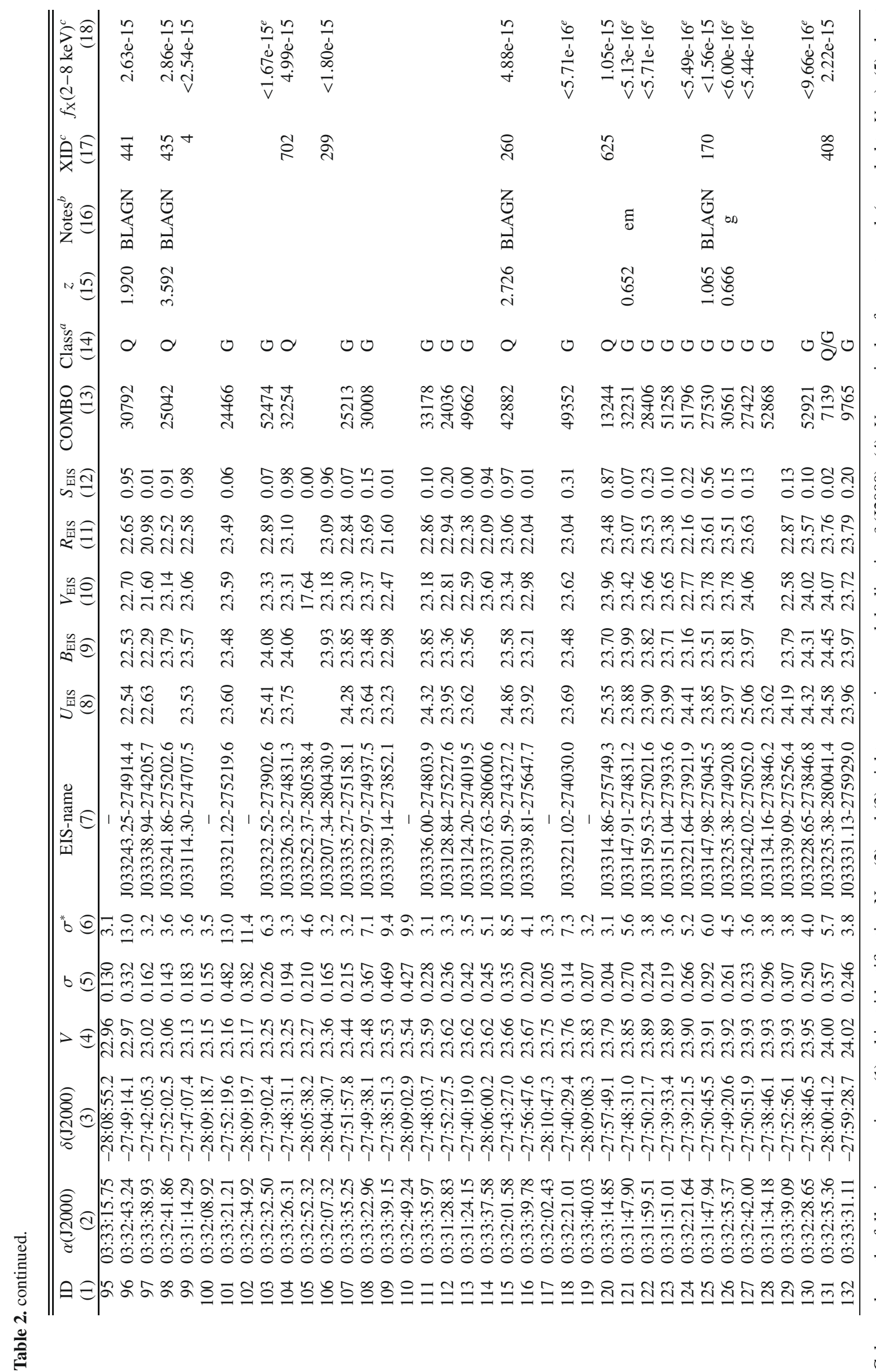



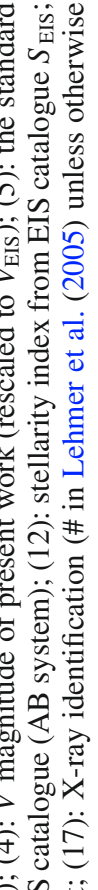

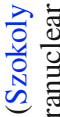

氙 育

胥

苛

동

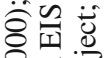

बㄹ हैं

$\infty_{0}^{\infty} \approx$

武

苍䨔

Z

닐

乙

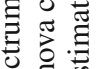

के

ธี

馬览

这出

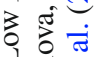

II $\overline{0}$

음

I क

的允允通

ठっ

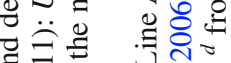

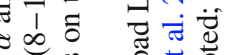

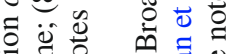

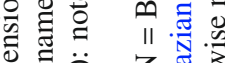

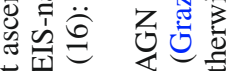

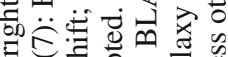

商苛

च ल

(눈

$\ddot{0}$ 휴

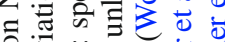

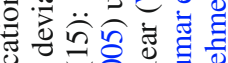

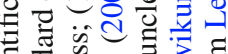

焉灾

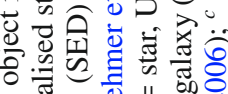

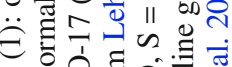

ن.

$\Xi \pm \sum, 0.0$

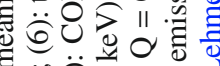

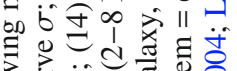

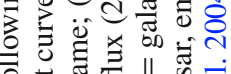

으

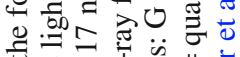

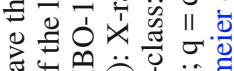

至

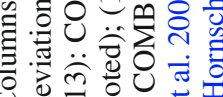

